\title{
ROTATING MACHINERY FAULT DIAGNOSIS BASED ON WAVELET FUZZY NEURAL NETWORK
}

\author{
Bin Peng ${ }^{1}$ Zhenquan Liu ${ }^{2}$ \\ I.College of Mechanical-Electronic Engineering, Lanzhou University of Technology, \\ Lanzhou 730050, China; \\ 2.College of Petrochemical Technology Lanzhou University of Technology, Lanzhou \\ 730050, China
}

Abstract: According to complicated fault characteristic of rotating machinery, its fault diagnosis based on wavelet fuzzy neural network (WFNN) which combines wavelet packet analysis and fuzzy neural network is put forward. By using it, the fuzzy fault diagnosis of rotating machinery is realized. All the arithmetic process of WFNN is realized through the computer. The results of simulation and test indicate that this method has obvious advantage for dealing with multi-coupled fault situation, the diagnosis method is simple, quick and has high correctness of fault diagnoses, proving that the diagnosis method is effective and providing a theoretical basis and new way for the fault diagnosis of rotating machinery.

Key words: Wavelet packet analysis, Fuzzy neural network, Rotating machinery, Fault diagnoses

Modern rotating machines are apt to appear faults because of their complexity and high speed. The complexity hampers the operator's ability to diagnose and eliminate equipment failures before their occurrence, so many systems are furnished with the intellectual monitoring system, fault diagnosis are the main embodiment of intellectual monitoring system, so the level of diagnose technology influences the function of intellectual monitoring system, it also has important meanings to keep normal running, reduce the product cost, raise product efficiency and ensure product safety for whole system. 
The fault diagnosis includes two steps: characteristic signal extraction and diagnose task. The first step is to draw the fault sign, the sign abstraction can adopt many kinds of techniques; the second step is really diagnose task which includes the type and severity of fault. Only the two steps have been confirmed, it could obtain overall fault description of rotating machinery. At present people carry on research just in a certain aspect and seldom combine the two sides to synthetically analyse, the text puts forward a new fault diagnoses method based on WFNN, all algorithms of WFNN are realized through the computer, WFNN can not only discern the correct type of fault but also get the serious level and development trend of fault[1].

\section{FAULT DIAGNOSIS OF ROTATING MACHINERY}

When rotating machinery appears fault, its vibration signal correspondingly changes, fault characteristics and types of vibration are reflected by these changed characteristic frequency band. The signal analyses is the main way as understanding equipment operation state and

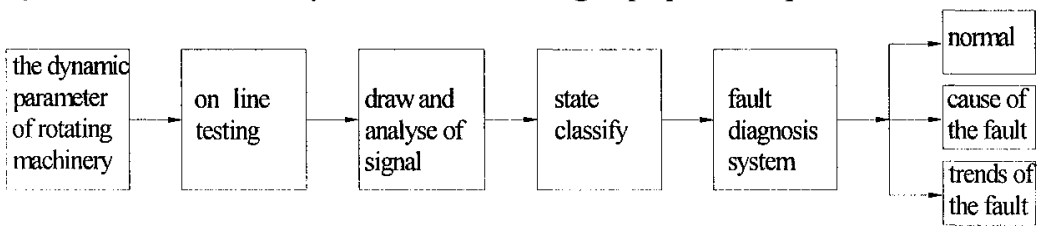

Figure 1. The systematic diagram of rotating machinery fault diagnosis

fault diagnosis, its main goal is to look for a kind of simple and effective signal transform method, it can show the important fault information and extract effective character signal. Loose WFNN combines wavelet packet analysis and fuzzy neural network, namely wavelet analysis signal is the input of fuzzy neural network, the types and development trend of fault can be gotten through the trained fuzzy neural network, Fig. 1 is the systematic diagram of rotating machinery fault diagnosis[2][3].

\section{WAVELET PACKET TRANSFORM}

Up to now, many kinds of signal transform have been applied to fault diagnose. Spectral analysis is the ripest method to draw signal characteristic of vibration, but FFT analyses have serious defects, firstly time information is lost while transforming from the time domain to spectral domain, FFT sets up on the basis of the steady signal, only suits to analyse steady signal. Secondly FFT is a kind of linearly method in essence, when the signal shows the strong nonlinearity, FFT can't deal with it, in addition anti-interference 
ability of FFT is relatively bad. Rotating machinery will be affected by various kinds of factors while running, it includes the non-steady composition in the output signals, especially when rotating machinery appears fault, its dynamics characteristic usually demonstrates complexity and non-linear, vibration signal also demonstrates nonstationarity, so it is very important to analyse this part signal. In order to overcome shortcoming of FFT, wavelet technology is put forward to deal with vibration signal and have made gratifying progress. The wavelet transform has the good characteristic in part, can realize the signal separation and don't lose any information. The wavelet transform can be applied to not only the steady signal but also the non-steady signal, so it is more suitable for vibration characteristic of signal than FFT. The article adopts wavelet packet to draw

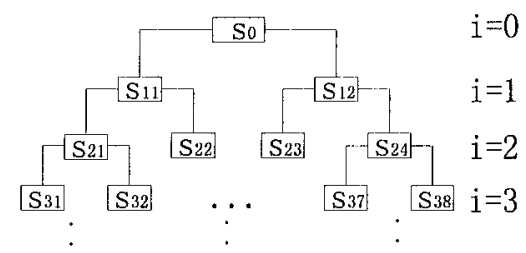

Figure2. The wavelet packet transform of signal

fault characteristic, wavelet packet is extension of wavelet, the defect of "a low resolving power of high frequency" is solved by wavelet packet transform, signal is divided into more careful frequency, different frequency weight has the corresponding node, those weights have different frequency characteristic, the relation of resolving coefficient is different too, the coefficient which relates to trouble information is kept among every node, interferential data are removed, it can reduce uproar, greatly compress the data, benefit real-time processing. Wavelet packet transform of signal is realized through Quadrature Mirror Filter, $s_{0}(t)$ is input signal, the transform expression is:

$$
\left\{\begin{array}{l}
s_{2 m}(t)=\sqrt{2} \sum_{n \in z} h(n) s_{m}(2 t-n) \\
s_{2 m+1}(t)=\sqrt{2} \sum_{n \in z} g(n) s_{m}(2 t-n)
\end{array} \quad t \in R \quad h(n) 、 g(n)\right. \text { express lowpass }
$$

and band-pass filter, function $\left\{s_{m}(t)\right\} m \in Z^{+}$is orthogonal wavelet packet. Wavelet packet transform is the fine time-frequency analysis of signal, it is the all frequency band decomposition result of primitive signal in all kinds of yardstick, it can effectively obtain time-frequency characteristic. Multistage decomposition signal about the original subset $\mathrm{s}$ can get through wavelet packet transform. It can realize the signal separation at different frequently band, it composes every frequency band of the resolved vibration signal, but these frequency bands are not all useful to discern the fault, it must filtrate characteristic frequency band, usually $0.5 f$ ( $f$ expresses work 
frequently), $f$ and multiple of $f$ have closer relation with fault, so these characteristic frequency bands are chosen as the characteristic frequency band. The energy value forms a vector in every frequency band, this vector has different value for different fault, therefore it can draw relevant fault information, offer a effective way for the fault diagnosis. Fig. 2 is the wavelet packet transform of the signal[4][5].

\section{FUZZY NEURAL NETWORK}

\subsection{The Structure of Fuzzy Neural Network}

The characteristic of extraction signal in wavelet packet is a vector which makes up of a lot of characteristic elements, the corresponding relation between it and fault is a kind of complicated nonlinearity mapping transformation, it is difficult to realize through the routine categorised method, the artificial neural network has unmatched conditions in this aspect,

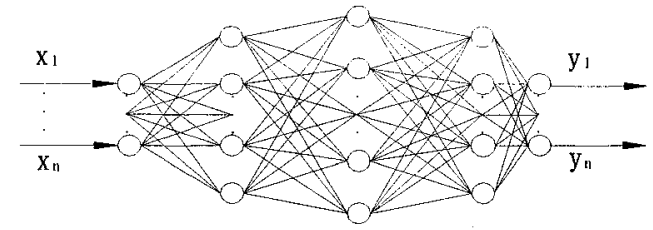

Figure 3. The structure of fuzzy BP neural network

it is a kind of ideal classification device. The second step of the fault diagnosis is a continuous mapping transformation course from fault sign vector $U$ to the fault reason vector $V$, the element of vector $V$ fetches continuous value between credibility area $[0,1]$. Because some faults or signs are fuzzy in rotating machinery, it is difficult to go on strict demarcation and can't use two value logic to describe and judge them briefly, at the same time the relation between some faults and signs is fuzzy, it is difficult to describe explicitly, so it can use the fuzzy law to realize continuous course of mapping transformation from sign vector $U$ to fault vector $V$. Fuzzy neural network which combines fuzzy logic system and neural network not only has the advantage of fuzzy message but also has the characteristic of selforganize and self-study of neural network. The fuzzy neural network can get the realistic fuzzy rule through studying and overcome the subjectivity of the fuzzy logic system in a certain extent, it will more suitable for the fault diagnosis of rotating machinery. The article uses a kind of serial structure fuzzy BP neural network, this network has five layers structure in Fig. 3. The first layer is input floor, input signal decides nodes of input layer, the main sign value can be confirmed according to the relation of shown signs and common fault of rotating machinery. The input value $x_{1}, x_{2} \ldots x_{n}$ don't change when reach the next layer in Fig.3. The second layer is to value input layer, 
Network

its function is fuzzy value. The third layer is imply floor, its node is confirmed according to the actual studying conditions in the network, imply layers apply sigmoid function, this layer node is used to realize mapping transformation from input variable fuzzy value to output variable fuzzy value, The fourth layer is output floor, every node represents one fault of rotating machinery, each fuzzy value represents fault degree, the fifth layer is weighting exported layer, it can realize clear output. It is apt to know that above five layers fuzzy neural network can realize mapping transformation from input to output, it can realize fault diagnosis for the input vector characteristic through the trained neural network.

\subsection{Membership Degree Function}

The main difference between fuzzy BP neural network and traditional BP neural network lies in its input and output expressing fuzzy membership degree, the network can deal with the digital input form and the semanteme input form through the proper membership degree function, its output is not a single categorised result but all kinds of membership degree. The key of fuzzy BP neural network is to ensure membership degree function, the distributed amplitude of every frequency is most sensitive to reflect the machine working state, so the input adopts the distributed amplitude of every frequency band in vibration frequency spectrum. For the semanteme form with value, the membership function can be expressed as trapezoid function, for the semanteme form with no value, the membership function can be expressed as:

(" $H$ " represents "very obvious", " $M$ " represents "obvious", " $L$ " represents "not obvious") $\quad H=\{0.05 / L ; 0.5 / M ; 0.95 / H\}$;

$M=\{0.5 / L ; 0.95 / M ; 0.50 / H\} ; L=\{0.95 / L ; 0.50 / M ; 0.05 / H\} ;$ if the input character value is numerical the membership function can be expressed as $\pi$ function, gauss function, in the practice project, different parts and points of machine have different vibration frequency spectrum, considering adaptability of network, membership degree function adopts the ratio of each frequency band amplitude in characteristic frequency band and total of amplitude, namely:

$$
\mu\left(x_{i}\right)=\frac{x_{i}}{\sum_{j=1}^{n} x_{j}} \quad i=1,2, \cdots, n
$$

in formula, $x_{i}, x_{j}$ represents spectral maximum of characteristic frequency band of $i$ and $j, n$ is the number of characteristic frequency band. 


\subsection{Fuzzy Neural Network Algorithm}

Traditional BP algorithm is a kind of simple steepest descent static behavior and seeking the excellent algorithm in fact, it will often make the learning process shake, convergence slowly. The first several steps are dropping very fast for the gradient algorithm, but with closing to optimum value, the gradient tends zero, it causes goal function drop slowly, but Newton rule can produce an ideal search direction near optimum value. In order to accelerate the speed of convergence of BP network, the article adopts Levenberg-Marquart (L-M) algorithm, L-M algorithm is the combination of gradient descent method and Newton method, its advantage lies in having both some characteristic of Newton method and overall characteristics of gradient method, it is convergence very fast when the weights of the network is small. Generally L-M method can obtain better result for most rotating machinery questions. Fault factor and fault exist fuzzy, so it is difficult to confirm the severity of the fault using accurate describe method, fuzzy discern offers the powerful means for solving this problem [6][7].

\section{APPLICATIONS}

In order to validate the effect of WFNN in fault diagnosis, the article compares WFNN and traditional BP neural network with a certain rotating machinery.

The vibration signal which gets from the sensor on the rotor laboratory system regards as the sample signal, signal is separated in every characteristic frequency band through wavelet packet transform, it is used to draw fault signal characteristic, the whole frequency band is divided into nine sections:

$0.01 \rightarrow 0.39 f, 0.4 \rightarrow 0.49 f, 0.5 f, 0.51 \rightarrow 0.99 f, f, 2 f, 3 \rightarrow 5 f$, odd,$>5 f \quad$. The article uses the vibration signal energy of nine frequency bands as diagnosing vector, while diagnosing, the output of network expressed the close degree of inputs vector and standard, namely it is the membership degree of various kinds of fault. The network input node is fault signs 9 , the fuzzy value layer node is 27 , output node is fault modes 5 , it can get the third layer node (imply layer) through trained and synthetically compared, latent node $\mathrm{h}$ is 10 in the article. Fuzzy BP neural network is trained according to $\mathrm{L}-\mathrm{M}$ algorithm, the stop error is 0.0001 . The test adopts the common fault of the rotating machinery: Oil-film Whirl fault, initial imbalance, rotor rubimpact, rotor bend, rotor crack, rotor misalignment and bearing house's looseness etc., 5 kinds of fault modes are summarized from a lot of fault 
cases, each kind of fault mode has been trained through fuzzy BP neural network, also trained through BP neural network. Through contrast, it proves that fault diagnosis ability of fuzzy neural network is higher than general BP

Table 1 . Fault input of rotating machinery

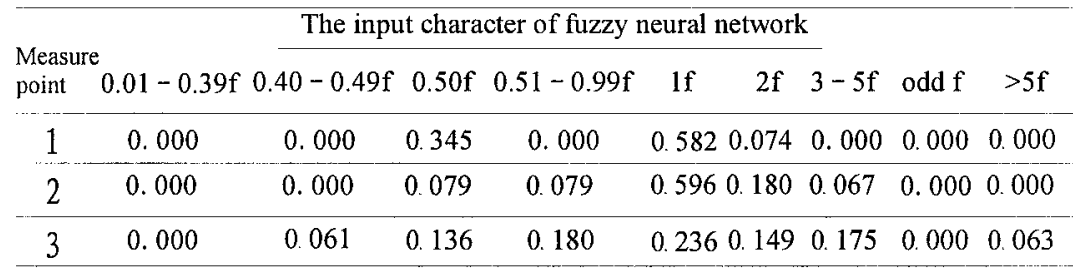

Table 2. The diagnosis result of WFNN

\begin{tabular}{cccccc} 
Measure point & $F_{1}$ & $F_{2}$ & $F_{3}$ & $F_{4}$ & $F_{5}$ \\
\hline 1 & 0.887 & 0.028 & 0.136 & 0.256 & 0.975 \\
\hline 2 & 0.930 & 0.261 & 0.001 & 0.962 & 0.184 \\
3 & 0.013 & 0.968 & 0.066 & 0.114 & 0.212
\end{tabular}

Table 3. The fault diagnosis result of BP neural network

\begin{tabular}{cccccc} 
Measure point & $F_{1}$ & $F_{2}$ & $F_{3}$ & $F_{4}$ & $F_{5}$ \\
1 & 0.101 & 0.002 & 0.003 & 0.005 & 0.879 \\
2 & 0.062 & 0.000 & 0.028 & 0.163 & 0.034 \\
\hline 3 & 0.000 & 0.805 & 0.025 & 0.000 & 0.014 \\
\hline
\end{tabular}

F1-F5 represent: Imbalance, Rub-impact, Rotor crack, Misalignment, Oil-film Whirl fault

neural network. Table 1 is the input fault of the rotating machinery, measure point 1 is very obvious imbalance fault and oil-film Whirl fault, measure point 2 is very obvious imbalance fault and misalignment fault, measure point 3 is very obvious rotor rub-impact fault, Table 2 is the diagnosis result of fuzzy neural network, it is very apt to find out that the fault diagnosis result is correct, can also get the developing direction of fault from the result. Table 3 is the fault diagnosis result of BP neural network, it can find out that only the third group fault diagnosis result of BP neural network is right, it also has mistake diagnosis (imbalance fault and misalignment fault) in the measure point 2. Its main reason is that BP network usually adopts the single fault sample to train, therefore it can not clearly or wrongly classify for many faults, but fuzzy BP neural network avoids this wrong through input 
and output membership degree to classify fault, the advantage is being more outstanding especially for dealing with multi-coupled faults.

\section{CONCLUSION}

The article puts forward a kind of new fault diagnosis method based on wavelet fuzzy neural network which combines wavelet packet analyse, fuzzy theory and neural network. It uses wavelet packet transform to draw the rotating machinery characteristic signal and offers the input characteristic of fuzzy neural network, it can get fault type and severe level according to the input characteristic through the trained fuzzy neural network, at the same time it also can get the developing direction of the output fault. The result of simulation proves that wavelet fuzzy neural network which integrates the merit of wavelet packet, fuzzy theory and neural network has very strong pattern-recognition and judgement as fault diagnosis method, it is a kind of extremely potential method in fault diagnosis of rotating machinery system.

\section{REFERENCES}

[1] Xu Feiyun, Jia Minping, Zhong Binglin, Huang Ren. Study on Fuzzy Neural Network for Fault Diagnosis of Rotating Machinery [J]. Journal of Vibration Engineering, 1996, $3(9), 21 \sim 219$.

[2] Zhang Ji-xian, Zhong Qiu-hai, Dai Ya-ping. Fault Diagnosis of Rotating Machinery Using RBF and Fuzzy Neural Network [J]. Journal of System Simulation, 2004, 16(3), $560 \sim 563$.

[3] HOU Xin-guo, XIA Li, WU Zheng-guo, ZHAO Yong-ling. Fault Diagnosis Method for Induction Motor Based on Wavelet Transformation and Neural Network [J]. Journal of Data Acquisition\& Processing, 2004, 19(1), 32 36

[4] Hou Xiaomei, Mao Zongyuan. The Application of Artificial Neural Network on Turbogenerators' Fault Diagnosis[J]. Journal of South China University of Technology (Natural Science), 1999, 27(8), 47 49

[5] Zhao Hong, Zhou Rui xiang, Lin Tingqi. Neural Network Supervised Control Based on Levenberg-Marquardt Algorithm [J].JOURNAL OF XI AN JIAO TONG UNIVERSITY. 2002, $36(5), 523 \sim 527$.

[6] Zhou Shouqin, Xie Youbai. Research on Machine Fault Diagnosis Based on Fuzzy Neural Networks [J]. Turbine Technology, 1999, 44 (4), $216 \sim 202$.

[7] Dong Tong. Study of Fuzzy Neural Network for Fault Diagnosis of turbine [J]. Techniques of Automation and Applications, 2001, 314 16. 